\title{
Is Routine Bilateral Neck Dissection Absolutely Necessary in the Management of NO CT Negative Neck in Patients with T4 Laryngeal Head and Neck Carcinoma?
}

\author{
Didier Dequanter ${ }^{1}$, Delphine Geukens ${ }^{2}$, Jean-Marie Bailly ${ }^{2}$, Mohammad Shahla ${ }^{1}$, Pascal Paulus ${ }^{3}$, \\ Philippe Lothaire ${ }^{3}$
}

${ }^{1}$ Department of surgery, 706 Rue de Gozée 6110 Montigny le Tilleul, Belgium; ${ }^{2}$ Department of radiology, 706 Rue de Gozée 6110 Montigny le Tilleul, Belgium; ${ }^{3} \mathrm{CHU}$ Charleroi, 706 Rue de Gozée 6110 Montigny le Tilleul, Belgium.

E-mail: didier.dequanter@pandora.be

Received Feburary 15 ${ }^{\text {th }}, 2011$; revised April 19 ${ }^{\text {th }}, 2011$; accepted May $17^{\text {th }}, 2011$.

\begin{abstract}
Objectives: Elective neck treatment of clinically N0 patients in patients with head and neck carcinomas is widely accepted as a standard approach. However, the issue whether elective neck treatment should routinely be directed on both sides of the neck is still controversial. The present study is aimed at determining whether T4 staged head and neck carcinomas required bilateral neck dissection in the management clinically No necks especially CT negative cervical nodes. Methods: We performed a retrospective analysis of patients with advanced head and neck disease who received bilateral neck dissection. All the patients had curative surgery as their initial treatment for the primary tumor and the neck. Results: All the 28 consecutive patients had T4 staged primary laryngeal cancer. Patients with clinically and radiologically $N+$ disease had invaded lymph node metastases in all cases. Patients staged clinically and radiologically NO had no invaded cervical lymph nodes found by pathologic examination. Patients staged clinically N0 and radiologically $\mathrm{N}+$ had invaded cervical lymph nodes in 8/12 cases and in 50\% (4/8) of the cases bilaterally. Conclusion: This study showed the importance on adequate clinical and radiological staging. By patients with advanced disease clinically and radiologically N0, bilateral neck dissection should not be necessary. But in patients radiologically $\mathrm{N}+$, routine bilateral neck dissection is beneficial.
\end{abstract}

Keywords: Advanced Head and Neck Cancer, Bilateral Neck Dissection, Radiological Evaluation

\section{Introduction}

Patients with advanced head and neck disease are at high risk for dissemination of the disease into cervical lymphatics, especially patients with advanced laryngeal cancers.

Indeed, the larynx has a rich lymphatic network that places patients with laryngeal carcinomas at high risk for nodal disease.

Therefore, elective neck treatment of clinically N0 neck in patients with carcinomas is widely accepted as a standard approach; however, the issue whether elective neck treatment should routinely be directed on both sides of the neck is still controversial.

The present study is aimed at determining whether T4 staged head and neck carcinomas required bilateral neck dissection in the management of clinically N0 necks, especially in CT negative cervical nodes.

\section{Materiel and Methods}

We performed a retrospective analysis of patients with advanced head and neck disease who had received bilateral neck dissection. All the patients had curative surgery as their initial treatment for the primary tumor and the neck.

\section{Results}

All the patients had a T4 laryngeal cancer. Clinical $\mathrm{N}+$ was accounted in $5 / 28$ patients.

All the 5 patients were also staged $\mathrm{N}+$ by radiological workup. The pathological examination showed lymph 
nodes metastases in all cases.

23 patients were staged clinically N0. Among these 23 patients, 11 patients were radiologically staged N0 and 12 patients were stage $\mathrm{N}+$.

All the patients staged clinically N0 and radiologically N0 (11/23) had no invaded cervical lymph nodes found by pathologic examination.

The patients staged clinically N0 and radiologically $\mathrm{N}+(12 / 23)$, had no lymph metastases in only 4 cases (4/12). In the majority of the cases, the pathological examination showed invaded lymph nodes (8/12) and in $50 \%$ of the cases (4/8) bilaterally.

\section{Discussion}

Bilateral neck treatment of clinically N0 neck patients with head and neck carcinomas is still controversial.

In their prospective study, Cagli et al. [1] evaluated, in their study, if supraglottic carcinomas require bilateral neck dissection in the management of N0 necks. They concluded that bilateral neck dissection may not be a part of the surgical procedure in all supraglottic laryngeal carcinomas patients (except in central tumors and lateral tumors with positive nodes at the ispilateral side of the neck.

Furthemore, they concluded, in their prospective study of previous untreated consecutive patients with clinically N0 neck laryngeal squamous cell carcinomas, that neck dissection may be unnecessary [2]

Similarly, Tu et all, in their study, found that there was not need for a comprehensive neck dissection for N0 neck in patients with supraglottic laryngeal cancer [3]. For Forlito [4], bilateral neck dissection, in cases of supraglottic cancer, may be necessary but only in patients with centrally or bilaterally located tumors.

Also, Gallo advocated bilateral neck dissection in supraglottic cancer particulary for advanced lesions extending or involving the midline larynx [5].

Kligeman [6] advocated bilateral neck dissection in T3/T4 laryngeal cancer patients. In their study, 56 patients were T3N0 and 20 patients were T4N0. All patients had bilateral neck dissection. The 5-year overall survival was $52 \%$. The disease free survival was $57 \%$.

In contrary, Alpert [7] concluded that routine neck dissection may not be necessary [5] in patients with supraglottic laryngeal cancers with bilaterally clinically uninvolved neck.

Neck dissection was performed on the involved neck in 36/52 node positive patients for either multiple involved nodes $(n=20)$ or size $>3 \mathrm{~cm}(n=16)$.

In patients with N0 neck in oral cavity squamous cell carcinomas, Koo et all advocated neck dissection only in patients with ipsilateral node metastasis or tumors, or both whose disease is greater than $\mathrm{T} 3$ or crossing the midline [8].

The same author advocated bilateral neck treatment only in patients with clinically ipsilateral node metastases and, in this study, in patients with pyriform sinus squamous cell carcinoma [9]. In patients with tonsillar squamous cell carcinoma they concluded that neck dissection could be performed only in patients with ipsilateral neck node metastasis [10].

Lim and Koo, in contrary, suggest bilateral neck dissection treatment in patients with oropharyngeal squamous cell carcinomas especially base of the tongue cancer. They proposed to include in the neck dissection neck levels II, III and IV instead of levels I, II and III [11].

Readelle de Zinis performed a selective neck dissection including levels II to IV whenever there is clinical or radiological evaluation of metastasis at any level [4] in patients with T2-T4 N0 oropharyngeal carcinomas [12].

In our study, in patients with advanced head and neck cancer, neck dissection was necessary and, guided by the radiological staging, in patients radiologically $\mathrm{N}+$. In patients staged clinically N0 and N+ radiologically, the radiological evaluation seems to be important before planning a bilateral neck dissection.

\section{Conclusions}

This study showed the importance on adequate clinical and radiological staging. By patients with advanced disease clinically and radiologically N0, bilateral neck dissection should not be necessary. In patients radiologically $\mathrm{N}+$, routine bilateral neck dissection is beneficial.

\section{REFERENCES}

[1] S. Cagli, I. Yce, O. G. Yigitbasi and E. Guney, "Is Routine Bilateral Neck Dissection Absolutely Necessary in the Management of N0 Neck in Patients with Supraglottic Carcinoma," European Archives of Oto-Rhino-Laryngology, Vol. 264, No. 12, 2007, pp. 1453-1457. doi:10.1007/s00405-007-0384-z

[2] Y. C. Lim, E. C. Choi, J. S. Lee, B. S. Koo, M. H. Song and H. A. Shin, "Is Dissection of Level IV Absolutely Necessary in Elective Lateral Neck Dissection for Clinically N0 Laryngeal Carcinoma,” Oral Oncology, Vol. 42, No. 1, 2006, pp. 102-107. doi:10.1016/j.oraloncology.2005.06.019

[3] G. Y. Tu, "Upper Neck (Level II) Dissection for N0 Neck Supraglottic Carcinoma,” Laryngoscope, Vol. 109, 1999, pp. 467-470.

[4] A. Ferlito, C. E. Silver and A. Rinaldo, "Selective Neck Dissection: A Rational Replacement for Complete Functional Neck Dissection in Patients with N0 Supraglottic and Glottic Carcinoma,” Head and Neck, Vol. 118, 2008, pp. 676-679.

[5] O. Gallo, I. Fini-Storchi and L. Napolitano, "Treatment of 
the Controlateral Negative Neck in Supraglottic Cancer Patients with Unilateral Node Metastases (N1-N3)," Head and Neck, Vol. 22, No. 4, 2000, pp. 386-392.

doi:10.1002/1097-0347(200007)22:4<386::AID-HED12> 3.0.CO;2-5

[6] J. Kligerman, L. O. Olivatto, R. A. Lima, E. Q. Freitas, J. R. Soares, F. L. Dias, L. E. Melo, G. M. Sa and E. Deuccini, "Elective Neck Dissection in the Treatment of T3/T4 N0 Squamous Cell Carcinoma of the Larynx," The American Journal of Surgery, Vol. 170, No. 5, 1995, pp. 436-439. doi:10.1016/S0002-9610(99)80324-9

[7] T. E. Alpert, S. Morbidini-Gaffney, C. T. Chung, J. A. Bogart, S. S. Hahn, J. Hsu and R. M. Kellmean, "Radiotherapy for the Clinically Negative Neck in Supraglottic Laryngeal Cancer," Cancer Journal, Vol. 10, 2004, pp. 335-338.

[8] B. S. Koo, Y. C. Lim, J. S. Lee and E. C. Choi, "Management of Controlateral N0 Neck in Oral Cavity Squamous Cell Carcinoma," Head and Neck, Vol. 28, No. 10, 2006, pp. 896-901. doi:10.1002/hed.20423

[9] B. S. Koo, Y. C. Lim, J. S. Lee, Y. H. Kim, S. H. Kim and E. C. Choi, "Management of Controlateral N0 neck In Pyriform Sinus Carcinoma,” Laryngoscope, Vol. 116, No. 7, 2006, pp. 1268-1272. doi:10.1097/01.mlg.0000225936.88411.71

[10] Y. C. Lim, S. Y. Lee, J. Y. Lim, J. S. Lee, B. S. Koo, S. H. Kim and E. C. Choi, "Management of Controlateral N0 Neck in Tonsillar Squamous Cell Carcinoma," Laryngoscope, Vol. 115, No. 9, 2005, pp. 1672-1675. doi:10.1097/01.mlg.0000184791.68804.0b

[11] Y. C. Lim, B. S. Koo, J. S. Lee, J. Y. Lim and E. C. Choi, "Distribution of Cervical Lymph Node Metastases in Oropharyngeal Carcinoma: Therapeutic Implications for the N0 Neck," Laryngoscope, Vol. 116, No. 7, 2006, pp. 1148-1152. doi:10.1097/01.mlg.0000217543.40027.1d

[12] L. O. Redaelli de Zinis, P. Nicolai, D. Tomenzoli, D. Ghizzardi, M. Trimarchi, J. Cappiello, G. Peretti and A. R. Antonelli, “The Distribution of Lymph Nodes Metastases in Supraglottic Squamous Cell Carcinoma: Therapeutic Implications," Head and Neck, Vol. 24, No. 10, 2002, pp. 913-920. doi.org/10.1002/hed.10152 